infection. Both pathogens target macrophages by evading innate immune recognition or intracellular killing. Since macrophages play a key role in inflammatory responses and their resolution, we tested the hypothesis that HIV-1 infection of macrophages may modulate inflammatory responses to co-infection with $\mathrm{Mtb}$ contributing to the immunopathogenesis of active tuberculosis (TB).

Methods Innate immune responses to Mtb were assessed in human macrophages with and without productive HIV-1 infection using genome-wide transcriptional profiling. Array data were validated by quantitative PCR and correlated with protein secretion in cell culture supernatants. The effects of Mtb co-infection on HIV replication were assessed by ELISA. The mechanisms underlying the observed phenotype were examined by Western immunoblotting and using selective inhibitors of innate immune signalling pathways.

Results HIV-1 infection of monocyte-derived macrophages leads to sustained, exaggerated pro-inflammatory responses to $\mathrm{Mtb}$ coinfection, including cytokines and chemokines that may recruit and activate further inflammatory leucocytes, and matrix metalloproteinases which play a role in tissue destruction. This phenotype is associated with rescue of HIV-1 replication following early repression in response to $\mathrm{Mtb}$. Our data suggest that augmented inflammatory responses to $\mathrm{Mtb}$ result from deficient induction of anti-inflammatory interleukin-10 in HIV-1 infected cells. None of these changes were evident in HIV-1 infected macrophages coinfected with Streptococcus pneumoniae, and the specificity of the effect in Mtb co-infection was mirrored by lower IL-10 and higher pro-inflammatory IL-1 $\beta$ in respiratory samples from HIV-1 infected patients with pulmonary TB compared to non-tuberculous respiratory infection. Complementation of deficient IL-10 responses to $\mathrm{Mtb}$ in HIV-1 infected macrophages reverses the exaggerated proinflammatory phenotype. HIV-1 infection attenuates phosphorylation of p38 and ERK1/2 in mitogen activated kinase pathways involved in IL-10 induction downstream of TLR2 and dectin-1 receptor stimulation. IL-10 production in HIV-1 infected cells is also inhibited in response to zymosan stimulation of these pathways. Inhibition of virus maturation with HIV-1 protease inhibitors, does not affect attenuation of IL-10 responses.

Conclusions Deficient induction of homeostatic IL-10 and consequent augmentation of pro-inflammatory responses may contribute to the immunopathogenesis of active $\mathrm{TB}$ and propagation of virus infection in HIV-1/Mtb co-infection.

\section{S131 HUMAN MACROPHAGE MODEL OF BIOMASS SMOKE EXPOSURE SHOWS IMPAIRED INGESTION OF STREPTOCOCCUS PNEUMONIAE}

doi:10.1136/thoraxjnl-2011-201054b.131

${ }^{1}$ A N Aljurayyan, ${ }^{2} \mathrm{D}$ G Fullerton, ${ }^{3} \mathrm{~S}$ Barrett, ${ }^{1} \mathrm{~S}$ B Gordon. ' Liverpool School of Tropical Medicine, Liverpool, UK; ${ }^{2}$ University Hospital Aintree, Liverpool, UK; ${ }^{3}$ University of Liverpool, Liverpool, UK

Background Three billion people worldwide, primarily in less economically developed countries use biomass fuel (BMF) as their main source of household energy. In children, the risk of pneumonia is increased by exposure to BMF smoke by a factor of 1.8. In adults, an epidemiological association between acute pneumonia and outdoor air pollution and tobacco smoke exposure has been demonstrated but there are no data confirming an association between pneumonia and BMF smoke exposure. Few mechanistic studies have been performed in humans investigating the association between BMF exposure and $S$ pneumoniae. This work aimed to assess the phagocytic ability of particulate matter (PM) challenged macrophages on fluorescent labelled beads and $S$ pneumoniae.

Methods In order to model BMF exposure, monocyte derived macrophages (MDMs), from 12 day old buffy coats, were challenged with different types and doses of PM (fine carbon black (FCB), Malawi wood and Norwegian wood). The percentage area of the macrophage cytoplasm that PM occupied (PM load) was calculated using image analysis software (Image SXM). The ability of PM challenged MDMs to phagocytose fluorescent labelled beads and $S$ pneumoniae was assessed by counting the beads / bacteria associated with MDMs, using fluorescent microscopy.

Results Increasing the PM dose was associated with a decrease in the percentage of cells associated with $S$ pneumoniae (Abstract S131 figure 1A) and beads. The same result was observed when the average number of $S$ pneumoniae (or beads) within cells was used as the outcome measure. With all three PMs used, a lower PM load was correlated with a higher capacity to phagocytose $S$ pneumoniae and beads. Malawi wood and Norwegian wood impaired MDMs phagocytic ability more than FCB (Abstract S131 figure 1B).
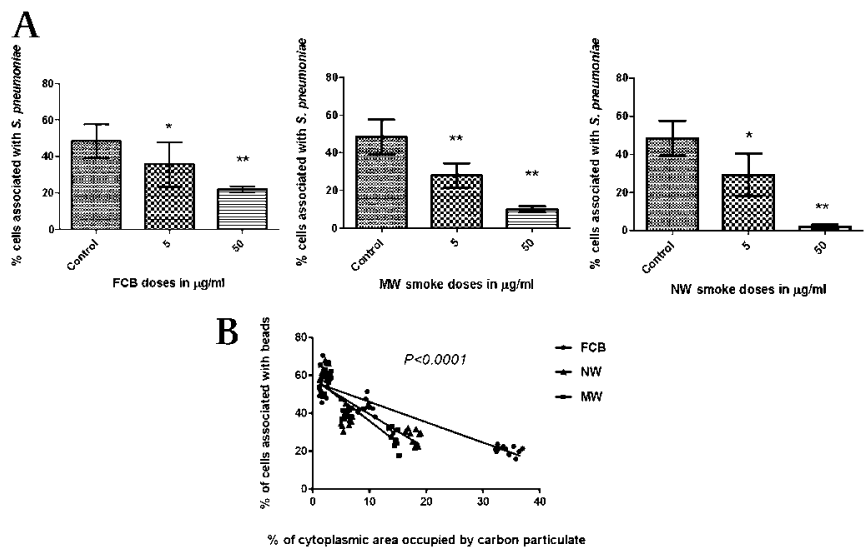

Abstract S131 Figure 1 (A) Comparison of percentage of cells associated with $S$ pneumoniae with 3 different types of particulate matter. (B) Comparison between the percentages of cells associated with $S$ pneumoniae and cytoplasmic area occupied by PM and three different types of PM (FCP, Norwegian wood and Malawi wood).

Conclusions Our data demonstrate that MDMs exposed to PM have impaired ability to phagocytose beads and $S$ pneumoniae and that wood smoke exposed MDMs had a greater phagocytic impairment than FCB. These observations support an association between BMF smoke exposure and pneumonia. Our model enables further work to be carried out on the dose-response of smoke exposure and pneumococcal disease as well as into the pathogenesis of increased susceptibility to pneumococcal infection in BMF exposed individuals.

\section{S132 INVESTIGATING THE ROLE OF PELLIN01, AN E3 UBIQUITIN LIGASE, IN MODULATING SIGNALLING PATHWAYS CONTROLLING THE INFLAMMATORY RESPONSE}

doi:10.1136/thoraxjnl-2011-201054b.132

J A Bennett, L R Prince, C A Stokes, L C Parker, M K Whyte, I Sabroe. University of Sheffield, Sheffield, UK

Viruses, such as Rhinovirus, are a major cause of asthma exacerbations. Pellino1 was identified as an interleukin 1 receptor associated kinase binding partner and has shown to act as an E3ubiquitin ligase that is involved in Lysine-63 polyubiquitination of interleukin 1 receptor associated kinase $1 / 4$ and RIP1, which are key mediators of the Toll-like receptor and interleukin 1 receptor (IL-1R) signalling pathways. As such, these pathways are implicated in responses to both bacterial and viral infections. However, the functional importance of Pellino proteins in regulating immune responses and their role in airway inflammatory diseases is yet to be 